\title{
A Parallel Block Iterative Method for the Hydrodynamic Device Model
}

\author{
Carl L. Gardner, Paul J. Lanzkron, and Donald J. Rose, Member, IEEE
}

\begin{abstract}
Block iterative methods are applied to hydrodynamic simulations of a one-dimensional (1-D) submicrometer semiconductor device. We show that block successive underrelaxation (SUR) converges with a fixed relaxation factor $\omega=$ 0.13 for simulations at $300 \mathrm{~K}$ and $\omega=0.04$ at $77 \mathrm{~K}$. To demonstrate the robustness of the block iterative method, we present numerical simulations of a steady-state electron shock wave in $\mathrm{Si}$ at $300 \mathrm{~K}$ for a $0.1-\mu \mathrm{m}$ channel and at $77 \mathrm{~K}$ for a $1.0-\mu \mathrm{m}$ channel. The block SUR method is parallelizable if each diagonal block solve can be done efficiently in parallel. Using chaotic relaxation and the preconditioned conjugate gradient method for the parallel diagonal block solves, we obtain a parallel speed up of approximately 2.5 on 10 processors of a Butterfly GP-1000. The 1-D simulations serve as a numerical laboratory for developing methods that will be essential for computational efficiency in two-dimensional (2-D) problems.
\end{abstract}

\section{INTRODUCTION}

$\mathrm{T}$ IIS investigation applies block iterative methods to both subsonic and transonic one-dimensional (1-D) hydrodynamic model computations. We show that block successive under-relaxation (SUR) converges with a fixed relaxation factor $\omega=0.13$ for simulations at $300 \mathrm{~K}$ and $\omega=0.04$ at $77 \mathrm{~K}$. The block SUR method is parallelizable if each diagonal block solve can be done efficiently in parallel. Using chaotic relaxation and the preconditioned conjugate gradient method for the parallel diagonal block solves, we obtain a parallel speedup of approximately 2.5 on 10 processors of a Butterfly GP-1000, a nonuniform memory access (NUMA) computer. Scaling arguments indicate that the block iterative method will be much more efficient than banded or sparse direct methods in two dimensions (2 D), and will parallelize much more efficiently. The 1-D simulations presented here serve as a numerical laboratory for developing methods that will be essential for computational efficiency in 2-D problems.

To demonstrate the robustness of the block iterative method, we present numerical simulations of subsonic flow and of a steady-state electron shock wave in a submicrometer semiconductor device, using a steady-state

Manuscript received July 3, 1990. The work of C. L. Gardner was supported in part by the National Science Foundation under Grant DMS8905872. The work of D. J. Rose was supported in part by the Office of Naval Research under Contract N00014-85-K-0487. This paper was recommended by Guest Editor M. Law.

C. L. Gardner and D. J. Rose are with the Department of Computer Science, Duke University, Durham, NC 27706.

P. J. Lanzkron is with the Departments of Computer Science and Mathematics, North Carolina State University, Raleigh, NC 27695.

IEEE Log Number 9101061. second upwind method. For the $n^{+}-n-n^{+}$diode (which models the channel of a MOSFET), the shock wave is fully developed in $\mathrm{Si}$ (with a $1-\mathrm{V}$ bias) at $300 \mathrm{~K}$ for a $0.1-\mu \mathrm{m}$ channel and at $77 \mathrm{~K}$ for a $1.0-\mu \mathrm{m}$ channel [1].

We give a brief introduction to the hydrodynamic model in Section II. In Section III, we describe the 1-D $n^{+}-n$ $-n^{+}$diode problem, and discuss the second upwind discretization of the 1-D steady-state hydrodynamic equations. The parallel block iterative method is described in Section IV. In Section V, we review central difference and second upwind computations for steady-state subsonic flow of electrons in the $n^{+}-n-n^{+}$diode. In Section VI, we review transonic electron flow computations in the $n^{+}-n-n^{+}$diode using the second upwind method. Section VI-6.1 presents a steady-state Mach 1.6 electron shock wave in Si at $300 \mathrm{~K}$, and Section VI-6.2 presents a steady-state Mach 4.1 electron shock wave in $\mathrm{Si}$ at $77 \mathrm{~K}$. We make some concluding remarks in Section VII.

II. The Hydrodynamic Model

The hydrodynamic model plays an important role in predicting the behavior of electrons in submicrometer semiconductor devices, since it exhibits hot carrier effects missing in the standard drift-diffusion model. The propagation of electrons in the semiconductor is approximated as the flow of a compressible charged fluid. This hydrodynamic approximation should be valid for devices with active regions $\geq 0.1 \mu \mathrm{m}$, since at this length scale simulations of the hydrodynamic model are in excellent agreement with Monte Carlo simulations of the Boltzmann equation [2]-[4]

The hydrodynamic transport equations were derived by Bløtekjær [5] from the Boltzmann equation:

$$
\begin{aligned}
\frac{\partial n}{\partial t}+\nabla \cdot(n \boldsymbol{v}) & =0 \\
\frac{\partial p}{\partial t}+\boldsymbol{v} \nabla \cdot \boldsymbol{p}+\boldsymbol{p} \cdot \nabla \boldsymbol{v}= & -e n \boldsymbol{E}-\nabla(n T)+\left(\frac{\partial p}{\partial t}\right)_{c} \\
\frac{\partial W}{\partial t}+\nabla \cdot(\boldsymbol{v W})= & -e n \boldsymbol{v} \cdot \boldsymbol{E}-\nabla \cdot(\boldsymbol{v} n T) \\
& +\nabla \cdot(\kappa \nabla T)+\left(\frac{\partial W}{\partial t}\right)_{c}
\end{aligned}
$$


where $n$ is the electron density, $v$ is the velocity, $p$ is the momentum density, $e(>0)$ is the electronic charge, $E$ is the electric field, $T$ is the temperature in energy units, $W$ is the energy density, $k$ is the thermal conductivity, and the subscript $c$ indicates collision terms. We will assume that the energy bands are parabolic:

$$
p=m n v, \quad W=\frac{3}{2} n T+\frac{1}{2} m n v^{2}
$$

where $m$ is the effective electron mass.

In addition to the transport equations (1)-(3), we have Poisson's equation for the electric field

$$
\nabla \cdot(\epsilon \nabla \phi)=-e\left(N_{D}-N_{A}-n\right), \quad \boldsymbol{E}=-\nabla \phi
$$

where $N_{D}$ is the density of donors and $N_{A}$ is the density of acceptors. Equations (1)-(5) determine the variables $n, p$, $W$, and $\phi$.

The nonlinear conservation laws (1)-(3) are the Euler equations [6] for a gas of charged particles in an electric field, with the addition of a heat conduction term. The polytropic gas constant $\gamma=5 / 3$, as is appropriate for a monatomic gas, and the soundspeed $c=\sqrt{T / m}$ [7].

Equations (1)-(3) are in conservation form, and may be written in terms of the variables $n, v, T$, and $\phi$. These variables represent the simplest choice for upwind methods.

$$
\text { III. The } n^{+}-n-n^{+} \text {Diode Problem }
$$

The $n^{+}-n-n^{+}$diode models the channel in a MOSFET, and clearly exhibits hot carrier effects at submicrometer scales. The diode begins with an $n^{+}$"source" region, is followed by an $n$ "channel" region, and ends with an $n^{+}$"drain" region.

We use the Baccarani-Wordeman models [8] for the collision terms and for the thermal conductivity:

$$
\begin{aligned}
\left(\frac{\partial \boldsymbol{p}}{\partial t}\right)_{c} & =\frac{-\boldsymbol{p}}{\tau_{p}} \tau_{p}=m \frac{\mu_{n 0}}{e} \frac{T_{0}}{T} \\
\left(\frac{\partial W}{\partial t}\right)_{c} & =\frac{-\left(W-\frac{3}{2} n T_{0}\right)}{\tau_{w}} \\
\tau_{w} & =\frac{m}{2} \frac{\mu_{n 0}}{e} \frac{T_{0}}{T}+\frac{3}{2} \frac{\mu_{n 0}}{e v_{s}^{2}} \frac{T T_{0}}{T+T_{0}} \\
\kappa & =\kappa_{0} \frac{\mu_{n 0}}{e} n T_{0}
\end{aligned}
$$

where $T_{0}$ is the ambient temperature, $\mu_{n 0}=\mu_{n 0}\left(T_{0}, N_{D}+\right.$ $\left.N_{A}\right)$ is the low field electron mobility, and $v_{s}=v_{s}\left(T_{0}\right)$ is the saturation velocity.

The simplest boundary conditions for the simulations are $n=N$ and $T=T_{0}$ at $x_{\min }$ and $x_{\max }$, and $e \phi\left(x_{\min }\right)=T$ $\ln \left(n / n_{i}\right)$ and $e \phi\left(x_{\max }\right)=T \ln \left(n / n_{i}\right)+e V$, where $V$ is the bias across the diode and $n_{i}$ is the intrinsic electron concentration.

The variables $n, T$, and $\phi$ are defined at the grid points $i=0,1, \cdots, N-1, N$, while the velocity $v$ is defined at the midpoints of the elements $l_{i-1 / 2}(i=1, \cdots, N)$ connecting grid points $i-1$ and $i$. The boundary conditions specify $n, T$, and $\phi$ at $i=0$ and $i=N$. Equations (1), (3), and (5) are enforced at the interior grid points $i$ $=1, \cdots, N-1$, while (2) is enforced at the midpoints of the elements $l_{i-1 / 2}, i=1, \cdots, N$. The discrete steadystate hydrodynamic equations are derived by using central differences (for subsonic flow) or the second upwind method (for subsonic or supersonic ${ }^{1}$ flow).

The second upwind method is a conservative discretization which captures the essential physical transport property of supersonic flow, that advected quantities directly influence the solution only downstream. For subsonic flow, the second upwind method corresponds to the Scharfetter-Gummel discretization for strong electric fields; for weak electric fields the Scharfetter-Gummel discretization corresponds to central differences [9].

The steady-state hydrodynamic equations in $1 \mathrm{D}$ have the form

$$
\left[\begin{array}{c}
f_{n} \\
f_{v} \\
f_{T} \\
f_{\phi}
\end{array}\right]=\frac{d}{d x}\left[\begin{array}{l}
v g_{n} \\
v g_{v} \\
v g_{T} \\
0
\end{array}\right]+\left[\begin{array}{l}
0 \\
h_{v} \\
h_{T} \\
h_{\phi}
\end{array}\right]+\left[\begin{array}{l}
0 \\
s_{v} \\
s_{T} \\
s_{\phi}
\end{array}\right]=0
$$

where

$$
\begin{aligned}
& g_{n}=n, \quad g_{v}=m n v, \quad g_{T}=\frac{5}{2} n T+\frac{1}{2} m n v^{2}-e n \phi \\
& h_{v}=-e n \frac{d \phi}{d x}+\frac{d}{d x}(n T), \quad h_{T}=-\frac{d}{d x}\left(\kappa \frac{d T}{d x}\right) \\
& h_{\phi}=\epsilon \frac{d^{2} \phi}{d x^{2}} \\
& s_{v}=\frac{m n v}{\tau_{p}} s_{T}=\frac{\frac{1}{2} m n v^{2}+\frac{3}{2} n\left(T-T_{0}\right)}{\tau_{w}} \\
& s_{\phi}=e(N-n) .
\end{aligned}
$$

In the second upwind method, the advection terms $d(v g) / d x$ in (9) are discretized using second upwind differences

$$
\frac{d}{d x}(v g)_{i} \approx\left(v_{i+(1 / 2)} g_{R}-v_{i-(1 / 2)} g_{L}\right) \Delta x
$$

where

$$
\begin{aligned}
& g_{R}= \begin{cases}g_{i} & \left(v_{i+(1 / 2)}>0\right) \\
g_{i+1} & \left(v_{i+(1 / 2)}<0\right)\end{cases} \\
& g_{L}= \begin{cases}g_{i-1} & \left(v_{i-(1 / 2)}>0\right) \\
g_{i} & \left(v_{i-(1 / 2)}<0\right)\end{cases}
\end{aligned}
$$

and central differences are used for $h_{v}, h_{T}$, and $h_{\phi}$.

\footnotetext{
'The central difference method is unstable for supersonic flow.
} 


\section{Block Iterative Methods}

We use Newton's method to linearize the discrete hydrodynamic equations (9):

$$
J\left[\begin{array}{l}
\delta n \\
\delta v \\
\delta T \\
\delta \phi
\end{array}\right]=-\left[\begin{array}{l}
f_{n} \\
f_{v} \\
f_{T} \\
f_{\phi}
\end{array}\right]=-f
$$

where $J$ is the Jacobian and $f$ is the 4 by 1 residual. The new solution is obtained by setting

$$
\left[\begin{array}{l}
n \\
v \\
T \\
\phi
\end{array}\right] \leftarrow\left[\begin{array}{l}
n \\
v \\
T \\
\phi
\end{array}\right]+t\left[\begin{array}{l}
\delta n \\
\delta v \\
\delta T \\
\delta \phi
\end{array}\right]
$$

where $t$ is a damping factor [10] between 0 and 1 , chosen to insure that the norm of the residual $f$ decreases monotonically.

The discretized Jacobian has the following block structure:

$$
J=\left[\begin{array}{cccc}
\frac{\delta f_{n}}{\delta n} & \frac{\delta f_{n}}{\delta v} & 0 & 0 \\
\frac{\delta f_{v}}{\delta n} & \frac{\delta f_{v}}{\delta v} & \frac{\delta f_{v}}{\delta T} & \frac{\delta f_{v}}{\delta \phi} \\
\frac{\delta f_{T}}{\delta n} & \frac{\delta f_{T}}{\delta v} & \frac{\delta f_{T}}{\delta T} & \frac{\delta f_{T}}{\delta \phi} \\
\frac{\delta f_{\phi}}{\delta n} & 0 & 0 & \frac{\delta f_{\phi}}{\delta \phi}
\end{array}\right]
$$

Each block is diagonal, bidiagonal, or tridiagonal. Newton's equation (15) may be solved directly by banded or sparse matrix techniques, or by block iterative methods.

Write Newton's equation (15) as

$$
J_{k} x=-f_{k}, \quad z_{k+1}=z_{k}+t_{k} x
$$

where $k=1,2, \cdots$ labels the Newton iterations, $x=$ $(\delta n, \delta v, \delta T, \delta \phi)^{T}, z=(n, v, T, \phi)^{T}$, and $t_{k}$ is a damping factor between 0 and 1 . The form of the Jacobian suggests solving Newton's equation by block iterative methods.

In $1 \mathrm{D}$, solving the discrete version of Newton's equation by banded methods is faster than using block iterative methods. However, in $2 \mathrm{D}$, block iterative methods will be essential, especially as the number of PDE's increases. Block iterative methods are ideally suited for the hydrodynamic model, since in $2 \mathrm{D}$ the number of PDE's is $K$ $=4 N_{\text {species }}+1$, where $N_{\text {species }}$ may be as large as four in GaAs (upper and lower valley electrons and holes). Solving $K$ PDE's on an $N \times N$ grid by sparse direct methods requires $O\left(K^{3} N^{3}\right)$ operations, while block iterative methods require $O\left(K N^{3}\right)+N_{\text {iterations }} \cdot O\left(K N^{2} \ln (N)\right)$ operations as $N$ and $K$ become large. The crossover from sparse direct to block iterative methods becomes favorable if $N_{\text {iterations }} \ll K^{2} N / \ln (N)$.

The number of "inner" iterative loops (versus "outer" Newton loops) is optimized by using the methods of [10]. We define

$$
\alpha_{k, n}=\left\|f_{k}+J_{k} x_{n}\right\|\left\|f_{k}\right\|^{-1}
$$

where $n=1,2, \cdots$ labels the inner iterations. If the iterative method converges, $\alpha_{k, n} \rightarrow 0$ as $n \rightarrow \infty$. We stop the inner iterations when

$$
\alpha_{k, n} \leq \alpha_{0}\left\|f_{k}\right\| /\left\|f_{0}\right\|
$$

where $\alpha_{0} \in(0,1)$. This criterion guarantees quadratic convergence of the Newton method. To actually attain quadratic convergence, the number of inner iterations must in general be squared at each successive Newton iteration. In practice, we place an upper bound on the number of inner iterations in exchange, possibly, for additional Newton steps.

One of our goals in this investigation is to find an efficient parallel algorithm for solving the hydrodynamic system (15) of equations. Chaotic relaxation would be an ideal parallel algorithm, since it gives linear speedup. (Chaotic relaxation is an algorithm based on the classical Jacobi and Gauss-Seidel relaxation schemes in which the solution point that is relaxed and the solution iterate used in the relaxation are arbitrary [11].) For the chaotic relaxation method to converge, the point Jacobi iteration

$$
D_{k}\left(x_{n}-x_{n-1}\right)=-\left(J_{k} x_{n-1}+f_{k}\right)
$$

must converge, where $D_{k}$ is the diagonal of $J_{k}$ and $n$ denotes the Jacobi iteration number. The point Jacobi iteration, however, does not converge for the 1-D steadystate hydrodynamic equations.

We found that block SUR does converge. Block SUR may be described by

$$
\left(D_{k}-\omega L_{k}\right)\left(x_{n}-x_{n-1}\right)=-\omega\left(J_{k} x_{n-1}+f_{k}\right)
$$

where $D_{k}$ is the block diagonal of $J_{k}, L_{k}$ is the block lower triangular part of $J_{k}$, and $\omega$ is the relaxation factor. If $\omega$ $<1$, the iteration is called under-relaxation.

In general (22) is computed sequentially and is not suited for parallel computers. A block solve must be computed of the form

$$
D_{i}^{k} x_{i}^{n}=r\left(x_{j>i}^{n-1}, \quad x_{j<i}^{n}\right)
$$

for every diagonal block $D_{i}^{k}$, where the subscripts $i$ and $j$ label the blocks, the superscript $k$ labels the outer Newton iterations, and the superscript $n$ labels the inner SUR iterations. In [12], Lanzkron showed that if each diagonal block solve can be done efficiently in parallel, sufficient speedup may be attained to make the whole iteration perform well. We then have two tasks: 1) to determine a blocking for $J_{k}$ for which block SUR converges, and 2) to parallelize the resulting diagonal block solves.

The equations provide a natural blocking. We found that the $\phi$ and $T$ equations in (22) may be separately blocked, but that the $n$ and $v$ equations must be consid- 
TABLE I

Timings on a SUN-4 IN MiNUTES: SECONDS FOR $N=60,120$, AND 240 Grid Intervals Using Banded and Block Iterative Solves. All Block Iterative Computations Were Done With $\omega=0.13$ for $300 \mathrm{~K}$ AND $\omega=0.04$ For $77 \mathrm{~K} . \alpha_{0}$ Was Set Equal to $1 / 2$ and The Maximum Number of InNER ItERations Was Set EQUal to $1 / 2 \mathrm{KN}$.

\begin{tabular}{lccc}
\hline Subsonic Flow & 60 & 120 & 240 \\
\hline banded & $0: 39$ & $1: 27$ & $3: 11$ \\
block iterative & $1: 11$ & $3: 39$ & $7: 29$ \\
\hline $300 K$ shock & 60 & 120 & 240 \\
\hline banded & $0: 45$ & $1: 41$ & $4: 01$ \\
block iterative & $1: 43$ & $4: 41$ & $12: 15$ \\
\hline $77 K$ shock & 60 & 120 & 240 \\
\hline banded & $2: 18$ & $4: 51$ & $10: 38$ \\
block iterative & $7: 02$ & $13: 16$ & $36: 00$ \\
\hline
\end{tabular}

ered as one large block. (No relaxation factor can be found to force convergence if the $n, v, \phi$, and $T$ equations are blocked separately.) This blocking is natural in that the $n$ and $v$ equations form a hyperbolic subsystem for the time dependent problem, while the $T$ and $\phi$ equations are parabolic and elliptic respectively.

In Table I, we show that the block SUR method converges for both subsonic and transonic problems, and compare block SUR with a banded matrix solve. Note that in $1 \mathrm{D}$ the equations may be re-ordered so that an 11 band system arises. The timings indicate that block SUR is competitive with banded solves in $1 \mathrm{D}$. This may seem counter intuitive, but there are two factors that make the block SUR computations relatively efficient: 1) the iterations need not solve the equations exactly ${ }^{2}$ (see $\left.[10]\right) ; 2$ ) only some of the rows in $J_{k}$ actually have 11 elements (others have only 4), and thus many extra operations are performed in the band factorization.

Efficient parallel solutions of the diagonal blocks are more difficult to obtain. The $T$ and $\phi$ equations are elliptic in steady state, and yield positive semi-definite systems. These equations converge under chaotic relaxation, and one can expect linear speedup for large systems. The $n-v$ block corresponds to a hyperbolic/elliptic system in steady-state, and various attempts at classical iterative methods have not yielded a parallelizable solution to this system.

The preconditioned conjugate gradient (PCG) method is a parallelizable method that converges for the $n-v$ block. Conjugate gradient (CG) is a semi-iterative technique for solving $A x=b$ that attemps to minimize the quadratic form $(1 / 2) x^{T} A x-b^{T} x$. There are several CG methods for nonsymmetric systems, e.g., CGS and BICG [13]. These methods are slow to converge or may even diverge if no preconditioning is performed. However, the standard incomplete LU factorization preconditioning for CGS does not lend itself to parallelization on NUMA architectures.

\footnotetext{
${ }^{2}$ In some cases, the number of Newton iterations required for global convergence increases.
}

Our approach is to modify the system and solve the symmetric system $A^{T} A x=A^{T} b$. As a preconditioner we use the diagonal of $A^{T} A$. Note that we do not need to explicitly form $A^{T} A$ since only matrix multiples are performed in CG. The diagonal of $A^{T} A$ can be formed in parallel. For the small systems we have considered up to this point, we have obtained speedups of approximately 2.5 on 10 processors of the Butterfly GP-1000.

\section{Computations for Subsonic Flow}

In this section and the next, we review the subsonic and transonic problems [1] simulated in Table I. The set of simulations is generic in that steady-state electron flow in the $n^{+}-n-n^{+}$diode may either involve shocks or not, and the two most important temperatures for semiconductor devices are 300 and $77 \mathrm{~K}$.

For the subsonic computations, we take a diode consisting of a $0.1-\mu \mathrm{m}$ source, a $0.4-\mu \mathrm{m}$ channel, and a $0.1-$ $\mu \mathrm{m}$ drain. In the $n^{+}$region, the doping density $N=5 \times$ $10^{17} \mathrm{~cm}^{-3}$, while in the $n$ region $N=2 \times 10^{15} \mathrm{~cm}^{-3}$. The ambient device temperature $T_{0}=300 \mathrm{~K}=0.0259$ $\mathrm{eV}$. The saturation velocity $v_{s}=1.03 \times 10^{7} \mathrm{~cm} / \mathrm{s}$.

To validate the second upwind method for subsonic problems, we performed simulations (with 120 grid intervals) of the $n^{+}-n-n^{+}$diode at $V=1 \mathrm{~V}$ using the hydrodynamic model.

Fig. 1 compares the temperature of the electron gas for the central difference and second upwind methods. Note that the temperature increases as the electrons flow from left to right across the channel. Also note the slight cooling of the electron gas as the electrons enter the channel. This cooling results from the electrons having to overcome a small potential barrier at the junction. In Fig. 2 we compare the electron velocity for the central difference and second upwind methods. Note the velocity overshoot phenomenon $v>v_{s}$ throughout most of the channel. Typical Mach numbers for these flows are $\leqslant 0.7$.

\section{Computations for Transonic Flow}

Higher electron velocities can be obtained in a semiconductor device by making the active device length shorter. Since the electron soundspeed $c=\sqrt{T / m}$, higher electron Mach numbers may also be obtained by lowering the ambient temperature.

We present two parameter regimes for the $n^{+}-n-$ $n^{+}$diode in which there is a transition from subsonic to supersonic electron flow just to the right of the $n^{+}-n$ junction and from supersonic to subsonic flow to the left of the $n-n^{+}$junction. In analogy with gas dynamical flow in a converging-diverging Laval nozzle, a shock wave develops at the transition from supersonic to subsonic flow. The $n^{+}-n-n^{+}$doping of the diode corresponds to the converging-diverging geometry of the Laval nozzle. For further analysis of the shock waves, see [1].

The transonic computations use the second upwind method. (The central difference method becomes unstable 


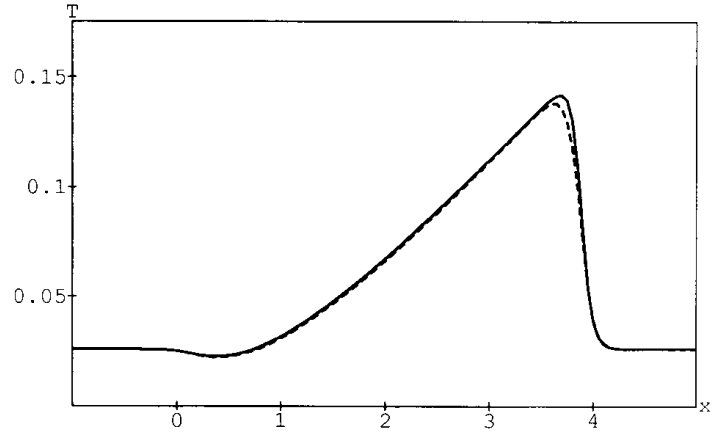

Fig. 1. Electron temperature in electronvolts for $V=1 \mathrm{~V}, 0.4-\mu \mathrm{m}$ channel, $300 \mathrm{~K}$ : central difference (solid) versus second upwind (dotted), $x$ is in $0.1 \mu \mathrm{m}$ for all figures.

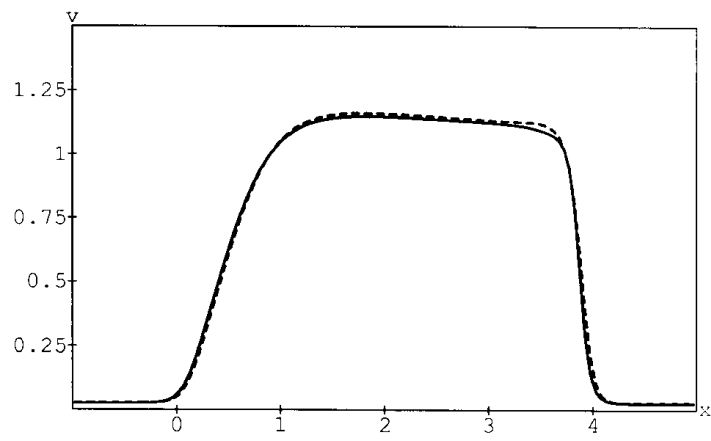

Fig. 2. Electron velocity in $10^{7} \mathrm{~cm} / \mathrm{s}$ for $V=1 \mathrm{~V}, 0.4-\mu \mathrm{m}$ channel, 300 $\mathrm{K}$ : central difference (solid) versus second upwind (dotted).

just as the maximum Mach number of the flow slightly exceeds 1.) The electron shock waves in Section VI-6.1 and 6.2 were originally simulated by Gardner [1] using the steady-state second upwind method, and have been reproduced by Fatemi, Gardner, Jerome, Osher, and Rose using a time-dependent nonoscillatory higher order upwind method in [14].

\subsection{Electron Shock Wave at $300 \mathrm{~K}$}

For the transonic computations at $300 \mathrm{~K}$, we take a diode consisting of a $0.1-\mu \mathrm{m}$ source, a $0.1-\mu \mathrm{m}$ channel, and a $0.1-\mu \mathrm{m}$ drain. In the $n^{+}$region, the doping density $N=5 \times 10^{17} \mathrm{~cm}^{-3}$, while in the $n$ region $N=2 \times 10^{15}$ $\mathrm{cm}^{-3}$.

Figs. 3 and 4 present a simulation of a Mach 1.6 electron shock wave at $V=1 \mathrm{~V}$ (with 120 grid intervals). Since the simulation is steady state, we are in the rest frame of the shock. The shock profile develops at $x_{s} \approx$ $0.035 \mu \mathrm{m}$ and is spread over about $5 \Delta x$. The electron flow is subsonic behind the shock profile and supersonic ahead of the shock profile. Note that the shock wave is a sharp profile in $v$ and $M$. The electron temperature is elevated well into the drain.

\subsection{Electron Shock Wave at $77 \mathrm{~K}$}

For the transonic computations at $77 \mathrm{~K}$, we take a diode consisting of a $0.1-\mu \mathrm{m}$ source, a $1.0-\mu \mathrm{m}$ channel, and a

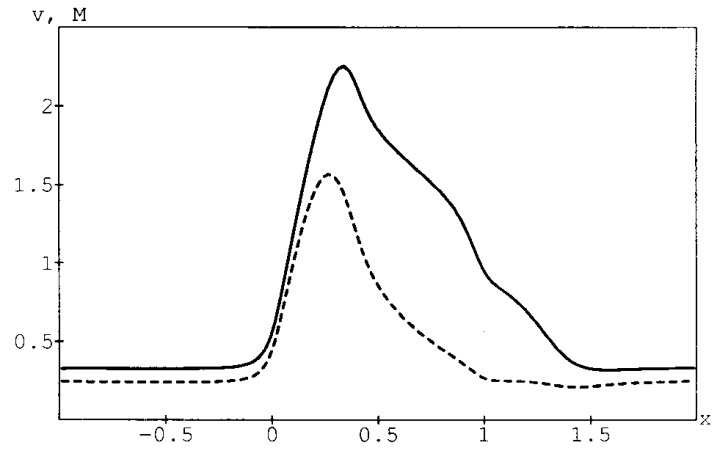

Fig. 3. Electron velocity in $10^{7} \mathrm{~cm} / \mathrm{s}$ and electron Mach number (dotted) for $V=1 \mathrm{~V}, 0.1-\mu \mathrm{m}$ channel, $300 \mathrm{~K}$.

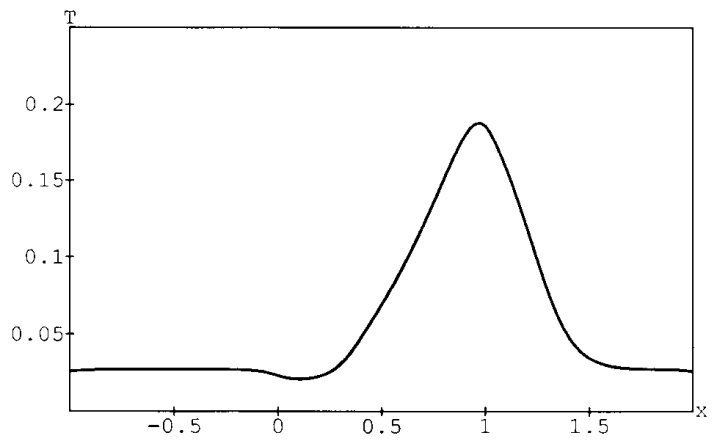

Fig. 4. Electron temperature in electronvolts for $V=1 \mathrm{~V}, 0.1-\mu \mathrm{m}$ channel, $300 \mathrm{~K}$.

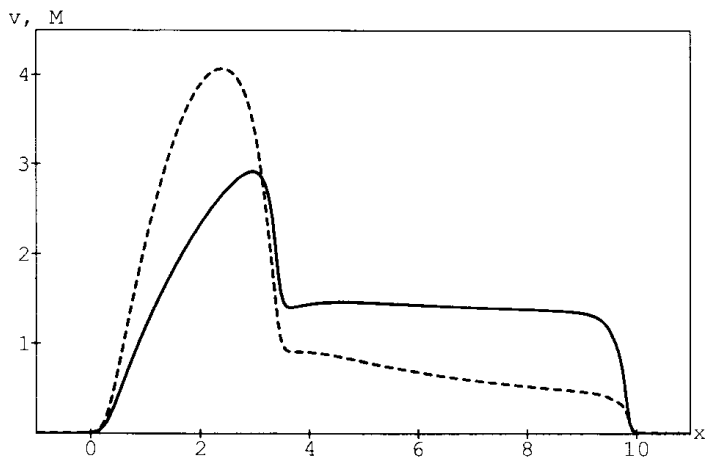

Fig. 5. Electron velocity in $10^{7} \mathrm{~cm} / \mathrm{s}$ and electron Mach number (dotted) for $V=1 \mathrm{~V}, 1-\mu \mathrm{m}$ channel, $77 \mathrm{~K}$.

$0.1-\mu \mathrm{m}$ drain. In the $n^{+}$region, the doping density $N=$ $10^{18} \mathrm{~cm}^{-3}$ (at $77 \mathrm{~K}$ ), while in the $n$ region $N=10^{15} \mathrm{~cm}^{-3}$ (at $77 \mathrm{~K}$ ). The ambient device temperature $T_{0}=77 \mathrm{~K}=$ $0.00665 \mathrm{eV}$. The saturation velocity $v_{s}=1.26 \times 10^{7}$ $\mathrm{cm} / \mathrm{s}$.

Figs. 5 and 6 present a simulation of a Mach 4.1 electron shock wave at $V=1 \mathrm{~V}$ (with 240 grid intervals). The shock profile develops at $x_{s} \approx 0.3 \mu \mathrm{m}$, and is spread over about $10 \Delta x$. Note that the shock wave is a much sharper profile in $v$ and $M$ than the Mach 1.6 shock wave. 


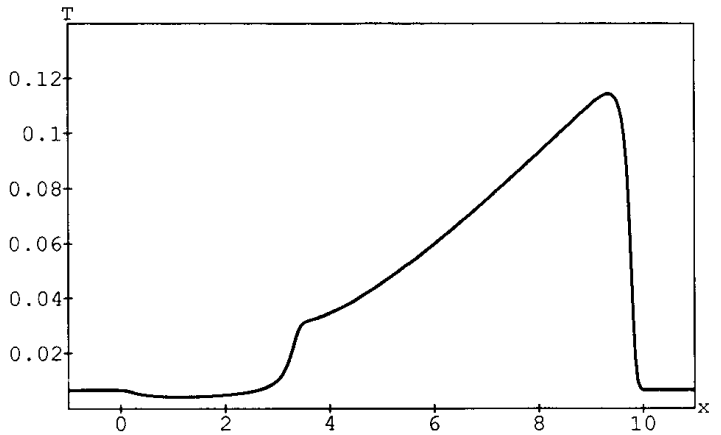

Fig. 6. Electron temperature in electronvolts for $V=1 \mathrm{~V}, 1-\mu \mathrm{m}$ channel, $77 \mathrm{~K}$.

\section{Conclusion}

We have demonstrated that block SUR converges for the hydrodynamic model for a 1-D submicrometer semiconductor device with a fixed relaxation factor $\omega=0.13$ for simulations at $300 \mathrm{~K}$ and $\omega=0.04$ at $77 \mathrm{~K}$. The same fixed relaxation factor works for both subsonic and transonic electron flow. This fact is suprising since in general the relaxation factor must change not only with each problem, but with each outer Newton iteration.

Parallel block iterative methods will be essential for computational efficiency in 2-D problems. For the 1-D $n^{+}$ $-n-n^{+}$diode, we obtained a parallel speedup of approximately 2.5 on 10 processors of a Butterfly GP-1000 using chaotic relaxation and the PCG method for the parallel diagonal block solves. There is far more communication between processors for PCG than for the chaotic relaxation method. We expect much better results for 2-D problems, since the hyperbolic/elliptic block will contain 2-D equations for $n$ and $v$, and the ratio of communication to computation will fall.

\section{REFERENCES}

[1] C. L. Gardner, "Numerical simulation of a steady-state electron shock wave in a submicrometer semiconductor device," IEEE Trans. Electron Devices, vol. 38, pp. 392-398, 1991

[2] F. Odeh, M. Rudan, and J. White, "Numerical solution of the hydrodynamic model for a one-dimensional semiconductor device," COMPEL, vol. 6, pp. 151-170, 1987

[3] M. V. Fischetti and M. Rudan, "Hydrodynamic and Monte Carlo simulation of an $n^{+}-n-n^{+}$submicron device," to be published.

[4] A. Gnudi, F. Odeh, and M. Rudan, "Investigation of non-local transport phenomena in small semiconductor devices," to be published.
[5] K. Bløtekjær, "Transport equations for electrons in two-valley semiconductors," IEEE Trans. Electron Devices, vol. ED-17, pp. 38-47, 1970.

[6] R. Courant and K. O. Friedrichs, Supersonic Flow and Shock Waves. New York: Springer-Verlag, 1948.

[7] C. L. Gardner, J. W. Jerome, and D. J. Rose, "Numerical methods for the hydrodynamic device model: Subsonic flow,"' IEEE Trans. Computer-Aided Design, vol. 8, pp. 501-507, 1989.

[8] G. Baccarani and M. R. Wordeman, "An investigation of steadystate velocity overshoot effects in Si and GaAs devices," Solid-State Electron., vol. 28, pp. 407-416, 1985.

[9] J. P. Kreskovsky, "A hybrid central difference scheme for solid-state device simulation," IEEE Trans. Electron Devices, vol. ED-34, pp. 1128-1133, 1987.

[10] R. E. Bank and D. J. Rose, "Global approximate Newton methods," Numer. Math., vol. 37, pp. 279-295, 1981.

[11] G. M. Baudet, "Asynchronous iterative methods for multiprocessors," J. Assoc. Comput. Mach., vol. 22, pp. 56-60, 1978.

[12] P. J. Lanzkron, "Convergence of nested iterative methods for linear systems," Ph.D. dissertation, Duke Univ., 1989.

[13] P. Sonneveld, "CGS, a fast Lanczos-type solver for nonsymmetric linear systems," SIAM J. Sci. Stat. Computing, vol. 10, pp. 36-52, 1989.

[14] E. Fatemi, C. L. Gardner, J. W. Jerome, S. Osher, and D. J. Rose, "Simulation of a steady-state electron shock wave in a submicron semiconductor device using high-order upwind methods," in Computational Electronics: Semiconductor Transport and Device Simulation. Boston, MA: Kluwer Academic, 1991.

Carl L. Gardner, for a photograph and biography, please see page 651 of the May 1991 issue of this Transactions.

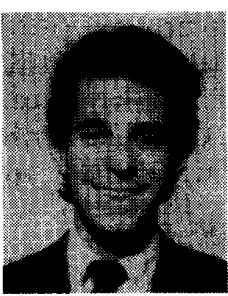

Paul J. Lanzkron received the B.S. degree in computer science from Northeastern University, Boston, MA, in 1984, and the M.S. and Ph.D. degrees in computer science from Duke University, Durham, NC, in 1987 and 1989 respectively. $\mathrm{He}$ is currently an Assistant Professor of Computer Science and Mathematics at North Carolina State University. His current research interests include VLSI circuit simulation, parallel methods for numerical linear algebra, and approximation methods for $N P$-complete problems. Dr. Lanzkron is a member of SIAM and ACM.

Donald J. Rose (M'89), for a photograph and biography, please see page 651 of the May 1991 issue of this Transactions. 